\title{
Valores humanos y voluntariado. Un estudio en personas mayores
}

\author{
Antonio Ariza-Montes ${ }^{1,2}$ (D), Pilar Tirado-Valencia ${ }^{1}$ (D), Vicente Fernández-Rodríguez ${ }^{1}$ (i) \\ ${ }^{1}$ Universidad Loyola Andalucia (Spain) \\ ${ }^{2}$ Universidad Autónoma de Chile (Chile) \\ ariza@uloyola.es,ptirado@uloyola.es,vicentef@uloyola.es
}

Received September, 2015

Accepted November, 2016

\section{Resumen}

Objeto: Esta investigación profundiza en el perfil de los valores humanos que influye en las personas mayores a la hora de colaborar de manera altruista como voluntarios. Para abordar la posible relación entre valores y voluntariado se adopta como perspectiva de análisis la Teoría de los Valores Humanos desarrollada por Schwartz (1992).

Diseño/metodología/enfoque: Tras un análisis exploratorio inicial, se plantea un modelo de regresión logística para determinar en qué medida el catálogo de valores personales que posee un individuo ayuda a explicar la actividad de voluntariado entre las personas mayores.

Aportaciones y resultados: Los resultados del modelo permiten afirmar que el hecho de que una persona mayor done o no tiempo a actividades de voluntariado puede ser satisfactoriamente explicado por el conjunto de valores humanos. Los resultados obtenidos revelan que los voluntarios jubilados presentan mayor sentido de la autotrascendencia y predisposición hacia el cambio, a la vez que denotan más aversión hacia la autopromoción y la conservación.

Implicaciones prácticas: Para mejorar el nivel de compromiso y la motivación de los voluntarios mayores jubilados, y afrontar con firmeza los retos que amenazan al sector no lucrativo, los responsables de los recursos humanos de las entidades del tercer sector deberán 
buscar la coherencia entre los valores individuales y la naturaleza de la actividad que va a desempeñar el voluntario, incrementando de este modo el grado de identificación de estas personas.

Originalidad / Valor añadido: A pesar de la abundante literatura que relaciona el voluntariado en personas mayores con el bienestar, tanto en términos de salud como de beneficios socioemocionales, y con las motivaciones de este colectivo a la hora de ejercer el voluntariado, no es frecuente hallar investigaciones que adopten como foco de análisis el perfil de los valores personales que incitan a estos individuos a colaborar con causas altruistas.

Palabras clave: Voluntariado, Valores humanos, Personas mayores, Sector no lucrativo

Códigos JEL: A13, D64, J49, L31

Title: Human values and volunteering. A study on elderly people

\section{Abstract}

Purpose: This research goes in depth in the human values profile that influences elderly to volunteer. In exploring possible relationships between values and volunteering, we adopt the analysis perspective of the Theory of Basic Human Values framework developed by Schwartz (1992).

Design/methodology: Based on an initial exploratory analysis, the study presents a logistic regression model that shows the extent to which an individual's personal values explain his/her volunteer behaviour on elderly people.

Findings: The results confirm that a retiree's engagement or lack of engagement in volunteer activities may be satisfactorily explained by the set of human values. The results show that retired volunteers experience a stronger sense of self-transcendence and predisposition towards change, and higher aversion to self-enhancement and conservation.

Practical implications: To increase the commitment and motivation of retired elder volunteers and to thus firmly confront challenges that threaten the non-profit sector, human resource professionals of non-profit organizations must ensure consistency between individual values and the nature of activities that volunteers perform, increasing such way those people's commitment. 
Originality/value: Despite the abundant studies that have related volunteering by elders to physical and socio-emotional wellbeing and to seniors' motivations while performing volunteer work, fewer studies have focused on personal values that prompt such individuals to participate in altruistic efforts.

Keywords: Volunteering, Human values, Elderly, Non-profit sector

Jel Codes: A13, D64, J49, L31

\section{Introducción}

En las últimas décadas el entorno socioeconómico ha experimentado profundos cambios. La sociedad actual requiere de la aparición de modelos de gestión capaces de integrar y dirigir una realidad cada vez más compleja, además de sistemas de colaboración coordinados donde la responsabilidad no recaiga casi exclusivamente en las autoridades públicas o privadas (Anshell \& Gash, 2008). Este nuevo contexto necesita de fórmulas de cooperación que involucren a entidades públicas -financiadas a través del sistema fiscal y presupuestario-, empresas privadas, que captan en el mercado los recursos necesarios para su funcionamiento, y organizaciones no lucrativas, cuyos recursos humanos y materiales proceden en gran medida de la solidaridad voluntaria de la ciudadanía. Precisamente este trabajo versa sobre uno de esos pilares, el tercer sector, que viene ganando protagonismo en el marco de la organización de la sociedad civil como actor principal, al expandir sus capacidades y abordar cada vez más esferas de actuación. El peso del sector no lucrativo en el conjunto de la economía mundial no ha dejado de crecer, significando a día de hoy una parte muy importante del tejido económico y social europeo. Según McKeever y Pettijohn (2014), en Estados Unidos existe casi un millón y medio de organizaciones no lucrativas que contribuyen con 887 billones de dólares a la economía estadounidense. Asimismo, uno de cada cuatro adultos en Estados Unidos colaboró con alguna organización de manera voluntaria durante 2013, aportando desinteresadamente 8.1 billones de horas de trabajo. En el contexto de la Unión Europea y refiriéndose exclusivamente al sector no lucrativo de la economía social, Ayensa (2011) apunta que este sector está formado por dos millones de organizaciones que representan el 10\% del empleo, en torno a 20 millones de trabajadores, y que suponen el 4\% del producto nacional bruto de la UE. Pese a la rotundidad que aportan tales magnitudes, para asimilar la relevancia del tercer sector hay que considerar otros aspectos intangibles de difícil cuantificación. Ayensa (2011) subraya tres de 
ellos. En primer lugar, la dimensión social del sector se ve reforzada al incorporarse la aportación del trabajo voluntario. En segundo lugar, el sector se caracteriza por ofrecer oportunidades de empleo a colectivos tradicionalmente desfavorecidos desde el punto de vista laboral como son las mujeres, los jóvenes o los discapacitados. Por último, muchas de las organizaciones del tercer sector prestan sus servicios a nivel local, capitalizando de este modo el tejido social más próximo.

El papel nuclear que juega hoy día el sector no lucrativo, tanto en el desarrollo económico como en el bienestar social, ha provocado que la literatura científica acerca de las motivaciones que inducen a una persona a dedicar parte de su tiempo a tareas de voluntariado sea extensa y rica. Este tema ha preocupado a investigadores de diferentes disciplinas: economistas, sociólogos, psicólogos,... Sin embargo, la gran mayoría de los estudios existentes, en cuanto al voluntariado y al origen de las donaciones canalizadas hacia el sector, tanto en tiempo como en dinero, se concentran casi de manera exclusiva en un sólo país: Estados Unidos. Las evidencias empíricas en otros países con respecto a los factores determinantes de las donaciones, son mucho menos frecuentes (Bauer, Bredtmann \& Schmidt, 2013). Este hecho provoca que las evidencias empíricas obtenidas en el ámbito europeo acerca de los valores que impregnan el ánimo de los voluntarios sean muy escasas, y más aún si se concentra la atención en un colectivo con particularidades muy concretas: las personas mayores retiradas ya del mercado laboral. Un alto porcentaje de voluntarios son mayores, y constituyen un importante recurso para resolver las necesidades de grupos vulnerables de diferentes edades (Keith, 2003). Sin duda, existen razones para presuponer que los patrones de voluntariado de las personas mayores son diferentes a los que dibujan los sujetos más jóvenes. Por un lado, desde la perspectiva de la Psicología del Desarrollo se defiende que la tasa de voluntariado es superior entre los mayores jubilados al disponer de más tiempo libre y aflorar en ellos sentimientos más altruistas (Piliavin \& Charng, 1990; Putnam, 2000). Este hecho está basado principalmente en la percepción de que las personas nacen egoístas y van evolucionando hacia perfiles más altruistas a través de la socialización y la educación (Haski-Leventhal, 2009). Por otro lado, desde una aproximación egocéntrica, algunos autores como Smith (2000) o Khalil (2004) consideran que el altruismo en estado puro no existe. Al contrario, todo acto de ayuda hacia los demás significa tan solo un mecanismo para la satisfacción propia. Desde este punto de vista, cualquier acción altruista se realiza en espera de algún beneficio o cooperación futura, y lo que motiva a una persona a ayudar a otra son los beneficios intangibles que esa persona espera obtener, ya sea directa o indirectamente. En esta línea, algunos estudios sobre el voluntariado en personas mayores han observado que los mayores parecen beneficiarse más del voluntariado que los más jóvenes (Van Willigen, 2000), posiblemente porque estas personas encuentran un mayor sentido a la vida a través del voluntariado (Greenfield \& Marks, 2004), al mismo tiempo que el voluntariado 
constituye un medio eficaz para construir nuevas redes sociales. Además, el voluntariado es promovido con frecuencia entre las personas más mayores de la comunidad como una forma de aportar un sentimiento de productividad y sentido durante el final de sus vidas (Martinson \& Halpern, 2011).

Adoptando como perspectiva de análisis la Teoría de los valores humanos de Schwartz (1992), en este trabajo se pretende indagar si existen unos valores personales en las personas mayores que activan el "gen" del voluntariado mientras que, por el contrario, otros lo neutralizan. Así pues, esta investigación profundiza en las motivaciones altruistas o egoístas, o bien el individualismo o conformismo, que llevan a ejercer el voluntariado a las personas que se encuentran en la última etapa de su vida. Este artículo comienza con una revisión de la literatura que estudia el voluntariado entre las personas mayores y su relación con la Teoría de los Valores Humanos. A continuación, describimos los datos del estudio y la metodología utilizada. Continuamos con la presentación de los resultados obtenidos en relación con nuestras hipótesis, y finalmente concluimos con una discusión sobre los resultados.

\section{Voluntariado, mayores y valores}

El potencial y la importancia del voluntariado en la última etapa de la vida ha recibido recientemente una atención creciente, tanto en el ámbito académico como en el político (Tang, Choi \& MorrowHowell, 2010). Hasta el momento son numerosos los estudios que profundizan en los efectos del voluntariado sobre el bienestar de las personas mayores y sobre su salud mental y física (Musick, Herzog \& House, 1999; Luoh \& Herzog, 2002; Morrow-Howell, Hinterlong, Rozario \& Tang, 2003; Lum \& Lightfoot, 2005; Hao, 2008; Morrow-Howell, Hong \& Tang, 2009; Ho, You \& Fung, 2012). Las investigaciones realizadas no sólo han podido demostrar los efectos positivos del voluntariado sobre las tasas de mortalidad y la calidad de vida entre los mayores, sino también sobre otros aspectos socioemocionales como el sentimiento personal de logro, el compromiso emocional y la socialización de estas personas, lo que les ayuda a contrarrestarlas pérdidas de todo tipo que inevitablemente ocurren a su edad (Hendricks \& Cutler, 2004).

Más allá del análisis de los efectos del voluntariado sobre la salud y los aspectos emocionales, también son frecuentes los estudios que indagan en las motivaciones que movilizan a los voluntarios mayores, así como en los factores explicativos que inciden en su mayor o menor identificación con la actividad que desempeñan. En este sentido, el estudio de Okun, Barr y Herzog (1998), realizado sobre una muestra de voluntarios con más de 50 años,demuestra que los elementos que más inciden en sus motivaciones son la posibilidad de expresar los valores propios a través del voluntariado, la 
participación en actividades que promueven la comprensión del mundo y la mejora de la autoestima. En un estudio posterior, Okun y Schultz (2003) concluyen que a medida que aumenta la edad decrecen las motivaciones relacionadas con la posibilidad de desarrollar una carrera profesional y de mejorar la comprensión del mundo y el aprendizaje. Pero que contrariamente a lo esperado, la edad no contribuye a predecir algunas otras motivaciones. La posibilidad de expresar los valores personales a través del voluntariado, la mejora de la autoestima o la reducción de sentimientos negativos son aspectos que incentivan el voluntariado sea cual sea la edad. Precisamente la expresión de los valores es el motivo más sobresaliente entre los voluntarios de este estudio. Asimismo, en una investigación realizada en Alemania, Italia y Holanda acerca de las motivaciones altruistas o egoístas de los voluntarios, Principi, Chiatti y Lamura (2012) no llegan a resultados concluyentes sobre la hipótesis de que las motivaciones altruistas incrementan con la edad, obteniendo resultados dispares en función del país y del sector de actividad en el que se desarrolle el trabajo voluntario.

Otros estudios demuestran que más allá de los aspectos personales, también hay cuestiones que inciden en la motivación que dependen de las características de la propia organización desde la que se ejerce la tarea de voluntariado. Tang et al. (2010) demuestran que el apoyo organizacional es un factor explicativo crítico de la motivación del voluntariado en personas mayores, ya que favorece el compromiso y la identificación,a la vez que maximiza los beneficios experimentados por estos voluntarios. Esta investigación pone de manifiesto que los beneficios son mayores cuanto menor es el estatus socioeconómico del individuo, medido a través de su nivel de educación e ingresos. En una línea similar, el estudio de Bang (2015) evidencia que la satisfacción en el trabajo tiene un efecto positivo más intenso en el deseo de continuar ejerciendo tareas de voluntariado entre los mayores que entre los jóvenes.

Pese a la abundante literatura que relaciona el voluntariado en personas mayores con el bienestar, tanto en términos de salud como de beneficios socioemocionales, y con las motivaciones de este colectivo a la hora de ejercer el voluntariado, lo que no es tan frecuente es hallar investigaciones que adopten como foco de análisis el perfil de los valores personales que incitan a estos individuos a colaborar con causas altruistas.

Los valores juegan un papel central en la conducta social, y cumplen un número importante de funciones en la vida de las personas. Esta afirmación es cierta hasta el punto de que Rokeach (1973) sugiere que el concepto de valor es capaz de unificar los intereses, aparentemente diversos, de todos los estudiosos del comportamiento humano. Los valores influyen en las actitudes y comportamientos cuando una persona se entrega en cuerpo y alma en el cuidado de un familiar enfermo, cuando dona 
dinero a una causa benéfica o cuando arriesga su propia vida tratando a pacientes de ébola de manera completamente altruista y desinteresada. Pero cuando un sujeto se dedica a otras actividades menos filantrópicas, más materialistas o incluso cuando delinque también lo hace guiado por unos valores que condicionan y dirigen su conducta. Esta circunstancia se produce porque los valores constituyen los criterios que las personas utilizan para evaluar las acciones, a las personas, y los acontecimientos. Los valores varían en importancia, pero los valores centrales de un individuo son los principios que le sirven de guía en su vida (Grönlund, 2012). Pese a enfrentarnos a una cuestión tan obvia, la realidad indica que existen pocas investigaciones que analicen en profundidad esta relación, fundamentalmente porque el estudio de los valores constituye un tema complejo debido a ciertas dificultades entre las que destacan (Hitlin \& Piliavin, 2004):

- los valores no son observables,

- las teorías actuales ayudan poco a entender cómo los valores moldean el comportamiento,

- las explicaciones sobre el comportamiento son poco convincentes cuando no se conoce el proceso de generación de valores,

- es difícil medir los valores,

- frecuentemente los valores están mezclados con otros fenómenos psicosociales, y

- los valores presentan variabilidad histórica y cultural en su contenido.

Todas estas dificultades están claramente relacionadas con el carácter intangible de los valores.

Las dos concepciones clásicas más aceptadas a la hora de definir qué son los valores son las de Kluckhohn (1951) -concepción, explícita o implícita, distintiva de un individuo o característica de un grupo, de lo deseable, la cual condiciona la elección entre los modos, medios y objetivos de una accióny la de Rokeach (1973)-un esquema racional que indica que las creencias, actitudes y valores de una persona deben estar en armonía entre sí, y que incluso determina cual es la importancia de esas creencias y valores a la hora de convencer a un individuo-. La primera enfatiza la acción. La segunda considera que los valores dan sentido a la acción.

Para abordar el asunto de la posible relación entre valores y voluntariado, en el presente trabajo se adopta como perspectiva de análisis la Teoría de los Valores Humanos desarrollada por Shalom Schwartz. Según Beckers, Siegers y Kuntz (2012), dos conceptos de valor han resultado dominantes en 
el campo de la investigación social: la Teoría de los Valores Humanos de Schwartz (incluida en la Encuesta Social Europea-ESS), y la teoría postmaterialista de Ronald Inglehart y su ampliación a los valores de autoafirmación (incluida en la Encuesta Mundial de Valores/Estudio Europeo de ValoresWVS/EVS). Schwartz (1992) define los valores como objetivos deseables, trascendentes, de importancia variable, que sirven de principios que guían la vida de una persona o de una entidad social (Schwartz, 1994). Schwartz (2006) ha resumido las principales características del concepto de valores básicos:

- los valores son creencias unidas estrechamente a sentimientos;

- los valores hacen referencia a objetivos deseables que motivan y orientan la actuación individual;

- los valores transcienden a situaciones y acciones concretas;

- los valores se utilizan como estándar o criterio de actuación, sirviendo así de guía para la elección de actos, políticas, personas y acontecimientos;

- los valores están ordenados por importancia, de tal manera que el conjunto de valores que impregna la esencia de todo ser humano constituye un sistema que lo caracteriza como individuo;

- la importancia relativa de los diferentes valores guía nuestra acción, hasta el punto de que cualquier actitud o comportamiento normalmente está condicionado por más de un valor.

La teoría de Schwartz sugiere la existencia de diez valores diferentes y universales. La idea sobre la que gira esta teoría es que en conjunto, todos ellos dibujan una estructura circular de valores motivacionales opuestos o compatibles, un modelo estructural que origina que las diferentes actuaciones que se derivan de algunos valores generen consecuencias psicológicas, prácticas y sociales que pueden ser congruentes o entrar en conflicto con el propósito derivado de otros valores distintos (por ejemplo, el universalismo y la benevolencia pueden ser compatibles, sin embargo, la benevolencia y el poder deberían ser incongruentes entre sî). Esta estructura circular representa un espacio motivacional continuo. En el modelo de Schwartz, cuanto más cerca se encuentran dos valores en cualquier dirección dentro del círculo, más parecida es la motivación asociada a dichos valores (Roszkowski, Kinzler \& Kane, 2014). Por consiguiente, a mayor distancia entre dos valores, más contraposición entre sus motivaciones. Esta estructura se resume en cuatro categorías de orden superior que dan lugar a dos grandes dimensiones bipolares. La primera dimensión (cambio-continuidad) enfrenta la apertura al cambio contra la 
conservación, mientras que la segunda dimensión (colectivismo-individualismo) contrapone la autotrascendencia con la autopromoción. Otros autores se han referido a estas dos dimensiones como individualismo versus conformismo y egoísmo versus altruismo (Held, Muller, Deutsch, Grzechnik \& Welzel, 2009). Por un lado, la autopromoción enfatiza la búsqueda de los intereses particulares y el logro del máximo potencial, mientras que la autotrascendencia implica la preocupación por el bienestar y los intereses del prójimo. Por otro lado, la apertura al cambio acentúa la acción y el pensamiento independiente, así como la predisposición hacia nuevas experiencias; sin embargo, la conservación exalta la autorregulación, el orden y la resistencia al cambio, en aras al mantenimiento y protección de lo que uno ya posee.

En la Figura 1 se presenta un resumen de cada uno de los diez valores fundamentales propuestos por Schwartz, así como los valores relacionados con cada uno de ellos.

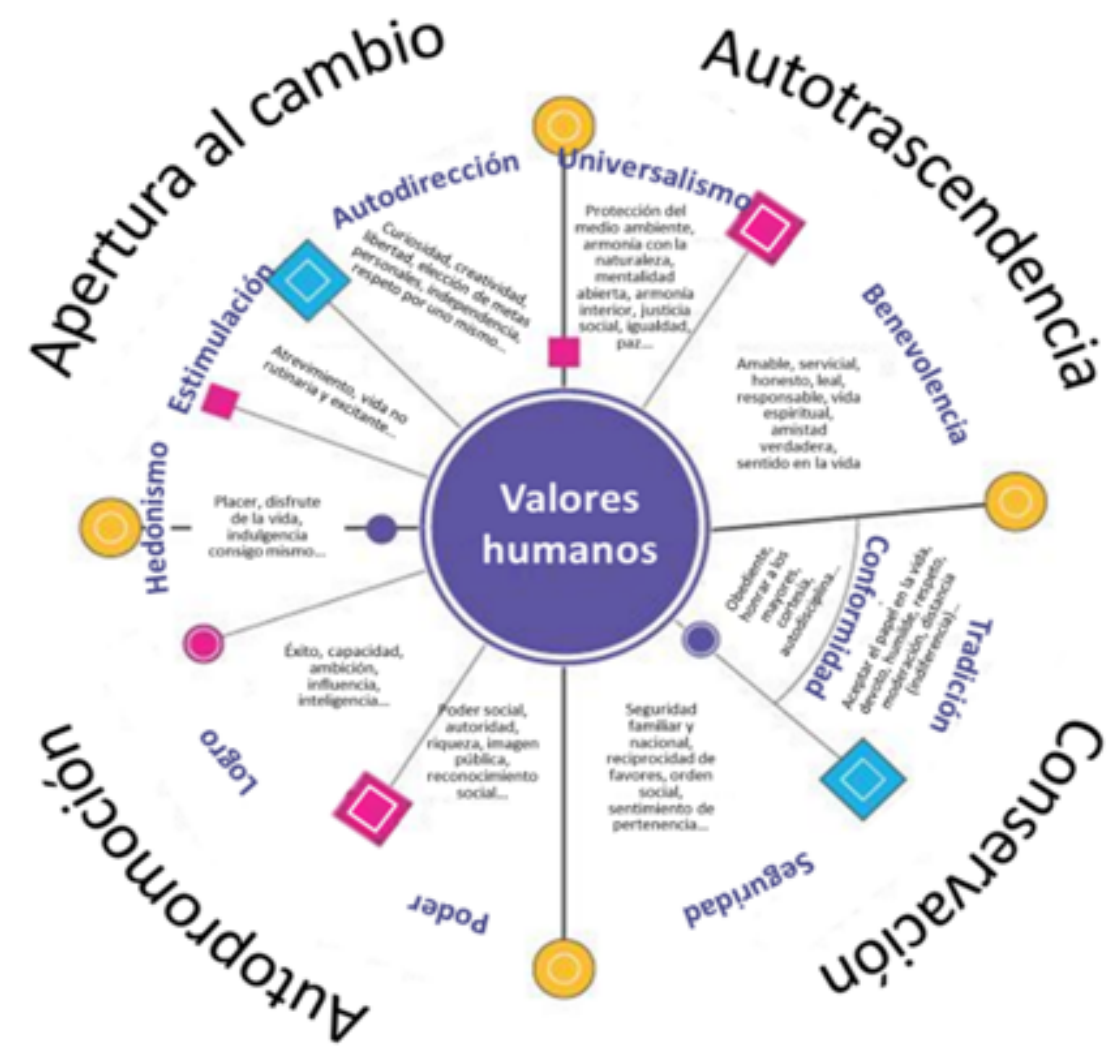

Figura 1. Estructura de la Teoría de los valores humanos de Schwartz (Schwartz, 1992)

Nota: El hedonismo comparte elementos de dos categorías distintas: la apertura al cambio y la autopromoción. 
La relación entre valores y voluntariado parece de sentido común. Sin embargo, en la literatura científica existen investigaciones que no han sido capaces de encontrar diferencias en cuanto a los valores personales entre los voluntarios y los no voluntarios (por ejemplo, Reed \& Selbee, 2003), o que en el mejor de los casos esta es muy débil (Wilson, 2000). Así y todo, autores como Dekker y Halman (2003) insisten en que los valores juegan un papel medular en el voluntariado.

En este sentido, el enfoque interno frente al externo y la orientación social frente a la personal constituyen algunas líneas de investigación relevantes en materia de voluntariado (Cnaan \& GoldbergGlen, 1991; Snyder \& Omoto, 1992; Clary, Snyder, Ridge, Copeland, Stukas \& Haugen, 1998; Yeung, 2004; Nylund \& Yeung, 2005; Musick \& Wilson, 2008). Aunque algunos autores como Clary y Snyder (1999) consideran que las motivaciones de los voluntarios tienen que ver tanto con la autotrascendencia (altruistas) como con la autopromoción (egoístas), lo más normal es reconocer a los valores altruistas como predictor esencial del voluntariado (Unger, 1991), hasta el punto de que el estudio de Moore, Warta y Erichsen (2014), realizado con una muestra de estudiantes universitarios, evidencia que estos valores suponen el motor más importante para la implicación en tareas de voluntariado. Asimismo, Bekkers y Bowman (2009) indican que es más probable que se involucren en el voluntariado las personas más confiadas y/o que consideran que ayudar a otros seres humanos constituye un importante objetivo vital. El altruismo, junto con el humanitarismo y la religiosidad, también son reconocidos como elementos fundamentales de la estructura motivacional del voluntariado en los trabajos de Bekkers (2005) y Musick y Wilson (2008).

De manera más específica, las investigaciones que utilizan la propia teoría de Schwartz sugieren que los valores relacionados con la autotrascendencia incitan al voluntariado, mientras que los de autopromoción lo obstaculizan. El propio Schwartz (2007) obtiene correlaciones positivas con el universalismo, al mismo tiempo que dicha relación se hace más débil, hasta el punto de transformarse en negativa, a medida que se aproxima a los valores de autopromoción personal. Este estudio revela que los valores individuales constituyen un predictor del voluntariado mucho más intenso que otras variables asociadas tradicionalmente al fenómeno como son la edad, el género o el nivel de ingresos, y que tan solo son superados por el factor educación. En un estudio posterior, Schwartz (2010) relaciona el voluntariado con la conducta prosocial, determinada según este autor por los valores del universalismo, la benevolencia y la conformidad. Así, mientras que la benevolencia y el universalismo serían motivos interiorizados para promover voluntariamente el bienestar de los demás, la conformidad tiene su fundamento en motivos externos que inducen a promover la conducta prosocial a fin de evitar consecuencias negativas en caso de no hacerlo. Por otra parte, Schwartz (2010) considera que el poder y 
la seguridad son valores que deberían obstaculizar la conducta prosocial, al comportar connotaciones egoístas. Con todo, cuando estos comportamientos conllevan el reconocimiento público y la aceptación social, los valores de la dimensión autopromoción, como el logro o el afán de poder, podrían promover el voluntariado más que obstaculizarlo (Schwartz, 2010; Roszkowski et al., 2014). Quizás esta dualidad de fuerzas - de atracción y de repulsión-es la que justifica que la investigación de Bathini y Vohra (2013) no obtenga una asociación significativa entre el valor del logro y la implicación en actividades de voluntariado.

En una línea de trabajo similar, el estudio llevado a cabo en Inglaterra por Pepper, Jackson y Uzzell (2009) sobre la conciencia social detecta, por un lado, correlaciones positivas de este fenómeno que debería fomentar el voluntariado con el universalismo y la benevolencia y, por otro, encuentra correlaciones negativas con el poder y el logro. De igual forma, la investigación de Sprecher y Fehr (2005) asocia el amor compasivo hacia los extraños con el valor del universalismo, identificándolo como un rasgo característico de las personas que realizan tareas de voluntariado. A una conclusión parecida llegan Mlčák y Záškodná (2014) al reconocer una dedicación al voluntariado más intensa entre los sujetos que presentan en mayor medida los dos valores del vector autotrascendencia, tanto la benevolencia como el universalismo. Según estos autores, estos individuos tienden de modo natural a comprender, aceptar y preservar el bienestar de sus congéneres. El modelo de ecuaciones estructurales planteado por Bathini y Vohra (2013) también concluye que el universalismo y la benevolencia se asocian con la participación en actividades de ayuda y voluntariado.

Utilizando datos de la Encuesta Social Europea, Plagnol y Huppert (2010) realizan un análisis de regresión logística que confirma la relación positiva del voluntariado con la benevolencia, por un lado, y con el universalismo, por otro. Además, de manera opuesta a lo que habían planteado como hipótesis de trabajo, el hedonismo y el logro también se relacionan positivamente con la participación en tareas de voluntariado. El estudio de estos autores sí que obtiene una asociación negativa entre poder y voluntariado, aunque esta no resulta estadísticamente significativa. Igualmente, un estudio en el contexto de la sociedad finesa, que analiza el papel del voluntariado como reflejo de los valores individuales y culturales, revela de manera concluyente que el universalismo fomenta el voluntariado (Puohiniemi, 2006). La relación negativa del voluntariado con ciertos valores del eje autopromoción en concreto con el poder y el hedonismo- también son identificados en los trabajos de Grönlund (2011, 2012).

$\mathrm{Si}$ bien los resultados de las investigaciones previas sobre la influencia de los valores de autotrascendencia y autopromoción en el voluntariado parecen bastante concluyentes, este mismo 
análisis necesita ser contrastado en el ámbito de las personas mayores. Por ello, este estudio propone el siguiente cuerpo de hipótesis de trabajo:

Hipótesis 1. Los valores de autotrascendencia -universalismo y benevolencia-presentan una asociación positiva con la participación en actividades de voluntariado de las personas mayores.

Hipótesis 2. Los valores de autopromoción -poder, logro y hedonismo- presentan una asociación negativa con el voluntariado de las personas mayores.

Si en el eje autotrascendencia versus autopromoción parece existir cierto consenso acerca de la relación con el voluntariado - positiva del primer vector y negativa del segundo-, al revisar los trabajos previos realizados sobre el eje apertura al cambio-conservación, las evidencias empíricas son más limitadas en cuanto ala cantidad de estudios y más difusas en lo que respecta a la calidad de los resultados obtenidos, sobre todo en lo que se refiere al vector conservación y sus valores asociados: seguridad, conformidad y tradición.

De este modo, el trabajo de Randle y Dolnicar (2015) -elaborado con una muestra de individuos que realizan tareas de voluntariado medioambiental- pone de manifiesto que estas personas conceden mayor importancia a ciertos valores, entre los que destacan la libertad, la creatividad, la disponibilidad, así como llevar una vida activa y emocionante, todos ellos relacionados con la dimensión apertura al cambio del modelo de Schwartz. Por otra parte, comparando una muestra de mujeres voluntarias con otra de mujeres que no se implican en este tipo de actividades, Mlčák y Záškodná (2014) comprueban que los voluntarios presentan puntuaciones más elevadas en los valores de autodirección y estimulación, tratándose de personas que prefieren la variedad de tareas y que tratan de evitar por todos los medios a su alcance el tedio y la rutina. Asimismo, la investigación de Bathini y Vohra (2013) obtiene una correlación positiva entre el valor estimulación y la implicación en actividades de voluntariado.

Por lo que respecta al eje conservación, tal y como se indicaba anteriormente, el propio Schwartz (2010) propone que el valor conformidad debería promover las conductas prosociales que favorecen el voluntariado, mientras que el valor seguridad debería atenuarlas. En esta línea argumental, la investigación de Mlčák y Záškodná (2014) detecta que personas que realizan voluntariado puntúan más bajo en el valor seguridad, en comparación con las que no se implican en estas actividades.

Dado que los resultados de las investigaciones previas sobre la influencia de los valores de apertura al cambio y conservación sobre el voluntariado no son tan concluyentes como en la dimensión de autotrascendencia-autopromoción, y puesto que estos resultados no se han contrastado aún en 
referencia al colectivo concreto de las personas mayores, a continuación se plantean las siguientes hipótesis de trabajo:

Hipótesis 3. Los valores de apertura al cambio -hedonismo, estimulación y autodirección - se relacionan positivamente con el voluntariado en las personas mayores.

Hipótesis 4. Los valores de conservación-seguridad, conformidad y tradición- se relacionan negativamente con el voluntariado en las personas mayores.

\section{Metodología}

\subsection{Recopilación de datos y muestra}

Los datos utilizados en esta investigación se han obtenido de la $6^{a}$ edición de la Encuesta Social Europea, realizada en 2012 y 2013 por un consorcio de investigación europeo conocido como ESSERIC. Esta encuesta proporciona información relacionada con las actitudes, las creencias y los patrones de comportamiento de diferentes poblaciones en 29 países europeos. A todas las personas con una edad igual o superior a 15 años, que viven en domicilios particulares, con independencia de su nacionalidad, lengua o estado civil se les preguntó sobre confianza social, preocupación política y participación, orientaciones político-sociales, exclusión social, lealtad nacional, étnica y religiosa, conocimiento y valoración de la democracia, bienestar personal y social, y finalmente, datos demográficos y socioeconómicos. El número total de entrevistados es superior a 44,000, habiéndose recopilado 28,420 encuestas en el año 2012 y 15,823 en el 2013.

A la hora de seleccionar la muestra se ha tenido en cuenta que cualquier análisis sobre voluntariado y valores debería considerar, por un lado, que las ratios de voluntariado cambian en función de la edad y/o las circunstancias sociolaborales de los encuestados y, por otra, que el perfil de valores de las personas evoluciona a lo largo del tiempo.

Para medir el nivel de voluntariado, se preguntó a los encuestados sobre la frecuencia con la que habían realizado algún trabajo para una organización caritativa o de voluntariado durante el año anterior. Aquellos sujetos que responden que nunca han realizado este tipo de actividades se codificaron como 0 , mientras que los individuos que declaran una frecuencia cuando menos trimestral o superior se clasificaron como 1. Con este criterio como punto de referencia, la ratio de voluntariado general que se obtiene de la Encuesta Social Europea es del 19.2\%. Esta ratio desciende hasta el 16.5\% entre los 
jóvenes de 15 a 29 años, se sitúa prácticamente en la media en el grupo de 30 a 45 años (19.1\%), alcanza un cénit del 20.9\% entre las personas de 46 a 64 años para, finalmente, situarse justo en la media entre el colectivo de personas que han cumplido 65 años o más (19.2\%). La situación sociolaboral de los encuestados también condiciona la tasa de voluntariado: el $27.1 \%$ de los ocupados con empleo remunerado realizan tareas de voluntariado; el 29.0\% de los estudiantes; el $25.3 \%$ de las personas que tienen como ocupación esencial las labores del hogar y el 15.4\% de los desempleados. Estas ratios contrastan con el $20.9 \%$ que muestran las personas que se encuentran jubiladas.

De un modo similar a lo que ocurre con las ratios de voluntariado según la edad o la situación sociolaboral, el compendio de valores que presentan las personas mayores, voluntarias y no voluntarias, difiere sustancialmente del que muestran otras cohortes de edad más jóvenes (ver Tabla 1). De este modo, a medida que aumenta la edad los valores relacionados con la autopromoción (poder, logro y hedonismo) y la apertura al cambio (hedonismo, estimulación y autodirección) van descendiendo, hasta alcanzar el nivel más bajo entre las personas mayores con 65 años o más. Al mismo tiempo van aumentando los sentimientos de conservación, observándose unos niveles más elevados, en tradición, conformidad y seguridad entre el grupo de personas de mayor edad. Finalmente, la autotrascendencia (universalismo y benevolencia) no presenta resultados diferenciados según el grupo de edad.

\begin{tabular}{|l|r|r|r|r|}
\cline { 2 - 6 } \multicolumn{1}{c|}{} & 15-29 años & 30-45 años & 46-64 años & 65 años o más \\
\hline Poder & 3.8 & 3.6 & 3.4 & 3.2 \\
\hline Logro & 4.5 & 4.2 & 3.8 & 3.6 \\
\hline Hedonismo & 4.6 & 4.1 & 3.9 & 3.5 \\
\hline Estimulación & 4.3 & 3.8 & 3.5 & 3.1 \\
\hline Autodirección & 4.8 & 4.7 & 4.6 & 4.4 \\
\hline Universalismo & 4.8 & 4.8 & 4.9 & 4.9 \\
\hline Benevolencia & 5.0 & 5.0 & 5.0 & 5.0 \\
\hline Tradición & 4.1 & 4.3 & 4.4 & 4.7 \\
\hline Conformidad & 4.0 & 4.1 & 4.2 & 4.5 \\
\hline Seguridad & 4.7 & 4.7 & 4.8 & 4.9 \\
\hline
\end{tabular}

Tabla 1. Valores de la teoría de Schwartz por grupos de edad

La búsqueda de una mayor homogeneidad muestral, que no esté condicionada ni por la edad de los encuestados ni por su situación sociolaboral, es la que nos ha llevado a extraer una submuestra de 3,214 individuos con 65 años o más que, además, estuvieran ya jubilados desde el punto de vista laboral. De estos, el 51.3\% reconocía no dedicarse nunca al voluntariado mientras que el 48.7\% lo hacían con cierta frecuencia, al menos una vez cada trimestre o más. La muestra se equilibró para evitar la tendencia del 
clasificador a incluir a todos los sujetos en la clase mayoritaria cuando existe cierto desequilibrio en el tamaño de las clases de aprendizaje.

De la muestra total, el 55.8\% son mujeres y el 44.2\% hombres. La tasa de feminización aumenta entre el grupo de personas que no participan en tareas de voluntariado $(57.8 \%)$ y se reduce entre los voluntarios (53.8\%). La edad media de los mayores se sitúa en 73.7 años, aumentando algo entre los no voluntarios (74.6 años) y descendiendo entre los voluntarios (72.9 años). Respecto al nivel de formación, el 24.6\% de los encuestados había cursado estudios primarios, el 46.7\% estudios secundarios y el $28.7 \%$ había finalizado estudios universitarios. La formación académica mejora entre los voluntarios (36.5\% de universitarios y sólo un 18.3\% de personas con estudios primarios), al mismo tiempo que se reduce entre los que no se implican en laborales altruistas $(21.4 \%$ de universitarios frente al $30.5 \%$ de sujetos con estudios primarios).

En índices iniciales, la priorización de valores de las personas que componen la muestra general de este trabajo es la siguiente: benevolencia (5.01), universalismo (4.91), seguridad (4.86) y tradición (4.63). La primera pregunta que nos surge es si este perfil difiere del de las personas que no realizan tareas de voluntariado. Los valores prioritarios para los jubilados voluntarios son la benevolencia (5.13), el universalismo (4.99) la seguridad (4.77) y la autodirección. Para los no voluntarios estos valores se ordenan del modo siguiente: seguridad (4.94), benevolencia (4.91), universalismo (4.83) y tradición (4.66).

\subsection{Metodología: El modelo de regresión logística binaria}

La metodología utilizada para el cumplimiento de los objetivos de este trabajo se fundamenta en el modelo de regresión logística binaria, un caso particular de los denominados modelos de regresión con respuesta dicotómica. Esta técnica estadística permite determinar la probabilidad de ocurrencia del suceso investigado -dedicar o no tiempo a la actividad de voluntariado entre las personas mayores en el caso que nos ocupa- frente a la probabilidad de ocurrencia del suceso contrario. 


\subsection{Medidas}

La revisión bibliográfica incluida en el apartado 2 proporciona la fundamentación para el diseño de la encuesta. Este estudio mide los valores sobre la base de la validación global y ampliada de la Teoría de los Valores Humanos de Schwartz. El método utilizado más frecuentemente en la investigación reciente sobre valores es la Encuesta de Valores de Schwartz (Lindeman \& Verkasalo, 2005). El instrumento inicial más popular para medir los diez valores propuestos por Schwartz fue un cuestionario de 40 ítems denominado "Portrait Value Questionnaire" (PVQ). Bajo la supervisión del propio Schwartz, la Encuesta Social Europea contiene una versión reducida del PVQ, que se utiliza regularmente para investigar las actitudes, creencias, y comportamientos en los países europeos. Este cuestionario utiliza cuatro dimensiones o valores de orden superior, los cuales contienen un total de 21 ítems. Esos ítems se combinan en 10 índices, uno para cada uno de los 10 valores fundamentales. Tres ítems miden el universalismo, y dos ítems miden cada uno de los otros valores básicos. La fiabilidad total de la escala utilizada en el cuestionario, medida a través del coeficiente alfa de Cronbach es de 0.822. Asimismo, se ha medido esta fiabilidad para cada una de las cuatro dimensiones de la estructura de valores, obteniendo unos coeficientes de confianza superiores a 0.7 en todos los casos (conservación $(\alpha=0.719)$, apertura al cambio $(\alpha=0.741)$, autotrascendencia $(\alpha=0.737)$ y autopromoción $(\alpha=0.724))$. Esta consistencia interna de los ítems de la escala es ligeramente superior a la presentada por Lindeman y Verkasalo (2005), quienes obtuvieron un coeficiente alfa de 0.60 para el eje conservación-apertura al cambio, y de 0.58 para el de autotrascendencia-autopromoción.

Las categorías de respuesta a esas preguntas forman una escala ordinal de seis puntos. Cada ítem presenta una descripción de un individuo, y entonces se pregunta al encuestado, en una escala bipolar asimétrica de 6 puntos, el grado en el que la descripción encaja con él (desde "en absoluto como yo"=6, a "muy similar a mi"=1).Con la intención de facilitar la interpretación de la escala, se adoptó la decisión de recodificar las opciones de respuesta para que oscilaran entre 1 (en absoluto como yo) y 6 (muy similar a mi), de tal modo que una puntuación más elevada signifique una mayor intensidad del valor al que esté referido el ítem en cuestión.

Por lo que respecta al voluntariado, tal y como se indicó anteriormente este trabajo considera que un voluntario es aquel individuo que se implica en tales tareas con cierta frecuencia, como mínimo trimestralmente; mientras que los no voluntarios serían aquellos sujetos que reconocen no haberse implicado nunca en estas labores durante los últimos 12 meses. Los primeros fueron codificados con un 1 y los segundos con un 0 . 


\section{Resultados}

\subsection{Análisis bivariante}

El objetivo general de este trabajo trata de explicar por qué algunas personas mayores participan en actividades de voluntariado mientras que otras no lo hacen. Para el estudio de las diferencias entre los subgrupos voluntarios-no voluntarios, se realizó la prueba de diferencias para muestra independientes T-Test. El propósito es examinar la posible relación bivariante entre la variable dependiente -estar implicado o no en actividades de voluntariado- y un conjunto de variables independientes que se corresponden con los diez valores propuestos por la teoría de Schwartz. Esta aproximación supone una preparación para el análisis multivariante que se realizará posteriormente, ya que en el modelo de regresión logística sólo se deberían incluir aquellas variables independientes que tienen una capacidad de predicción estadísticamente significativa.

En la Tabla 2 se presentan las puntuaciones medias obtenidas para cada uno de los diez valores propuestos por la teoría de Schwartz, tanto a nivel de la muestra general incluida en la Encuesta Social Europea, como en la submuestra empleada en la presente investigación. El análisis de diferencia de medias revela que existen tales diferencias estadísticamente significativas entre voluntarios y no voluntarios en ocho de los diez valores analizados. En los únicos en los que no se producen tales diferencias son en el logro y la tradición, por lo que ambos serán excluidos del análisis multivariante posterior.

\begin{tabular}{|c|c|c|c|c|c|}
\hline \multirow[t]{2}{*}{ Valor } & $\begin{array}{c}(1) \\
(N=43,559)\end{array}$ & $\begin{array}{c}(2) \\
(\mathrm{N}=3,136)\end{array}$ & $\begin{array}{c}(3) \\
(\mathrm{N}=1,545)\end{array}$ & $\begin{array}{c}(4) \\
(\mathrm{N}=1,591)\end{array}$ & \multirow[t]{2}{*}{$\operatorname{Sig}\left(^{(*)}\right.$} \\
\hline & $M(S D)$ & $M(S D)$ & $M(S D)$ & $M(S D)$ & \\
\hline Poder & $3.48(1.11)$ & $3.09(1.08)$ & $3.02(1.06)$ & $3.15(1.09)$ & 0.000 \\
\hline Logro & $3.99(1.19)$ & $3.52(1.23)$ & $3.54(1.21)$ & $3.49(1.24)$ & 0.255 (n.s.) \\
\hline Hedonismo & $4.00(1.19)$ & $3.62(1.26)$ & $3.87(1.16)$ & $3.38(1.31)$ & 0.000 \\
\hline Estimulación & $3.62(1.22)$ & $3.12(1.18)$ & $3.34(1.16)$ & $2.91(1.16)$ & 0.000 \\
\hline Autodirección & $4.66(0.94)$ & $4.52(1.02)$ & $4.76(0.89)$ & $4.28(1.07)$ & 0.000 \\
\hline Universalismo & $4.85(0.77)$ & $4.91(0.76)$ & $4.99(0.71)$ & $4.83(0.79)$ & 0.000 \\
\hline Benevolencia & $5.01(0.79)$ & $5.01(0.81)$ & $5.13(0.76)$ & $4.91(0.84)$ & 0.000 \\
\hline Tradición & $4.38(1.03)$ & $4.63(0.97)$ & $4.60(0.96)$ & $4.66(0.97)$ & 0.093 (n.s.) \\
\hline Conformidad & $4.22(1.09)$ & $4.47(1.06)$ & $4.42(1.09)$ & $4.52(1.04)$ & 0.007 \\
\hline Seguridad & $4.78(0.99)$ & $4.86(0.98)$ & $4.77(1.00)$ & $4.94(0.95)$ & 0.000 \\
\hline
\end{tabular}

(1) Población general de la Encuesta Social Europea ( $>15$ años). (2) Población objeto de estudio ( $\geq 65$ años y jubilada). (3) Mayores voluntarios. (4) Mayores no voluntarios.

* Diferencia significativa para un nivel de confianza del 95\%. n.s.: no significativo.

Tabla 2. Valores de la teoría de Schwartz por grupos poblacionales 


\subsection{Análisis multivariante}

Tras el análisis exploratorio inicial, a continuación se plantea un modelo de regresión logística para determinar en qué medida ayudan a explicar la actividad de voluntariado el catálogo de valores personales que posee un individuo. Para estimar el modelo se ha optado por el método por pasos adelante RV, usando todas las variables predictoras para evaluar qué combinación es la más eficiente en la explicación de la dedicación al voluntariado entre los jubilados con 65 años o más.

Así, tal y como se puede constatar en el modelo multivariante cuyos resultados se exponen en la Tabla 3, el hedonismo, la estimulación, la autodirección y la benevolencia son los valores personales que contribuyen positivamente al voluntariado entre los mayores jubilados. Al mismo tiempo, el poder y la búsqueda de la seguridad personal se asocian negativamente con el ejercicio de dicha actividad. Todos los resultados presentados son significativos en el nivel del 1\%. Para este nivel de significación, el modelo de regresión logística indica que ni el universalismo ni la conformidad se relacionan - ni positiva, ni negativamente- con la implicación en actividades de voluntariado entre las personas de más edad, por lo que estos dos valores han sido eliminados del modelo.

A pesar de todo, el impacto de cada una de las variables significativas en la probabilidad de dedicar tiempo al voluntariado difiere sustancialmente de unas a otras, tal y como indica el análisis de los intervalos de confianza obtenidos para las correspondientes odds ratios (ver Tabla 3). En sentido positivo, el efecto más importante se aprecia en el valor personal de la autodirección (OR: 1.494), con un intervalo de confianza que oscila entre 1.364 y 1.636. A ello se une que la probabilidad de ser voluntario aumenta entre las personas más imbuidas del valor de la estimulación (OR: 1.134; IC: 1.0481.227) y del hedonismo (OR: 1.218; IC: 1.133-1.309), confirmándose de este modo una relación positiva con el voluntariado de los tres valores que conforman la dimensión apertura al cambio. Por su parte, los OR correspondientes al valor benevolencia (1.332) de la dimensión autotrascendencia -con un intervalo de confianza que oscila entre 1.191 y 1.489- sugieren que las personas mayores más benevolentes presentan mayor probabilidad de dedicación al voluntariado.

En sentido negativo, la obsesión por el poder reduce la probabilidad de dedicar tiempo a tareas altruistas y desinteresadas (OR: 0.769; IC: 0.712-0.831), algo previsible dada la pertenencia de este valor individual a la dimensión autopromoción. Asimismo, las personas que valoran ante todo su propia seguridad es menos probable que se impliquen en actividades de voluntariado (OR: 0.703; IC: 0.6430.770), confirmándose de este modo la relación negativa de esta variable perteneciente a la dimensión conservación del modelo de Schwartz. 
El estadístico de contraste aplicado para evaluar la validez del modelo en su conjunto indica que existen razones suficientes para aceptar la validez del mismo (la prueba ómnibus del modelo, utilizada para este fin, presenta los siguientes resultados: Chi Cuadrado: 366.709; Sig. 0.0000), es decir, para afirmar que el hecho de que un jubilado done o no tiempo personal a actividades de voluntariado puede ser satisfactoriamente explicado por el conjunto de valores recogidos en la Teoría de los Valores Humanos de Schwartz. Asimismo, la capacidad de generalización del modelo resulta aceptable, ya que el 63.5\% de los individuos considerados resultó bien clasificado conociendo de antemano su situación real. Además, existe un buen equilibrio entre los porcentajes correspondientes a voluntarios $(63.0 \%)$ y no voluntarios (64.1\%), lo que confiere mayor fiabilidad y garantía al modelo. Aunque como es sabido existen otros factores al margen de los valores humanos que contribuyen a explicar la implicación en actividades de voluntariado -sobre este tema se puede consultar el trabajo de Ariza-Montes, Roldán-Salgueiro and Leal-Rodríguez (2015)-, los valores humanos por sí solos presentan una buena capacidad para predecir la participación o no en actividades de voluntariado por parte de las personas mayores.

\begin{tabular}{|c|c|c|c|c|c|c|c|}
\hline & \multicolumn{4}{|c|}{ Variables en el modelo } & \multicolumn{3}{|c|}{$\begin{array}{c}\text { Odds ratios } \\
\text { I.C. } 95 \% \text { para OR }\end{array}$} \\
\hline & B & E.T. & Wald & Sig. & OR & Inferior & Superior \\
\hline Poder & -0.262 & 0.039 & 44.676 & 0.000 & 0.769 & 0.712 & 0.831 \\
\hline Hedonismo & 0.197 & 0.037 & 28.472 & 0.000 & 1.218 & 1.133 & 1.309 \\
\hline Estimulación & 0.126 & 0.040 & 9.762 & 0.002 & 1.134 & 1.048 & 1.227 \\
\hline Autodirección & 0.401 & 0.046 & 75.049 & 0.000 & 1.494 & 1.364 & 1.636 \\
\hline $0,00 €$ & 0.102 & $67,00 €$ & 2.294 & 0.130 (n.s) & 1.107 & 0.970 & 1.264 \\
\hline Benevolencia & 0.287 & 0.057 & 25.318 & 0.000 & 1.332 & 1.191 & 1.489 \\
\hline Conformidad & -0.022 & 0.042 & 0.264 & 0.607 (n.s.) & 0.979 & 0.091 & 1.063 \\
\hline Seguridad & -0.352 & 0.046 & 58.977 & 0.000 & 0.703 & 0.643 & 0.770 \\
\hline Constante & -1.883 & 0.287 & 42.947 & 0.000 & 0.152 & & \\
\hline$\chi^{2}$ Test de eficiencia & 368.709 & & & & & & \\
\hline Grados de libertad & 6 & & & & & & \\
\hline Nivel de significación & 0.000 & & & & & & \\
\hline$\%$ correcto de predicción & & & & & & & \\
\hline Global & $63.5 \%$ & & & & & & \\
\hline Voluntarios & $63.0 \%$ & & & & & & \\
\hline No voluntarios & $64.1 \%$ & & & & & & \\
\hline
\end{tabular}

Tabla 3. Regresión logística: factores que determinan el voluntariado en mayores e intervalos de confianza de los odds ratios 


\section{Discusión y conclusiones}

El voluntariado cubre diferentes funciones, necesidades y objetivos en la vida de las personas. En algunos casos, el voluntariado constituye un mecanismo de autorrealización personal, hasta el punto de generar efectos positivos tanto para los voluntarios como para los receptores de sus desvelos. Entre otros beneficios, Burns, Reid, Toncar, Anderson y Wells (2008) destacan el aprendizaje de nuevas habilidades o la socialización. Si a esta circunstancia se une el elevado número de personas que colaboran con organizaciones del tercer sector en Europa, conocer cuáles son los factores que condicionan los niveles de participación e implicación de estos individuos se erige en un asunto de gran importancia. No obstante, se trata de una cuestión sumamente compleja, dada la variedad de interpretaciones que adquieren las motivaciones y las variables socioculturales que inciden sobre el perfil del voluntario, analizadas tanto a nivel individual (persona a persona), como en términos colectivos (asociaciones y redes de solidaridad).

Entre las motivaciones que inducen a una persona a destinar parte de su tiempo a una actividad de voluntariado, que sin duda son complejas, con muchos matices y aristas, la literatura enfatiza la influencia de los valores y de las creencias en el voluntariado (Wilson, 2000). El estudio de la relación valores-voluntariado ha sido una cuestión abordada desde diferentes disciplinas como la sociología, la psicología, la economía, la gestión de recursos humanos o la organización empresarial, entre otras, sin llegar a resultados concluyentes en esta materia (Inglehart, 1997; Hofstede, 2001; Grönlund et al., 2011).

El presente trabajo se enmarca precisamente en este contexto tan etéreo: el de los valores intangibles que dirigen la conducta de los voluntarios. Para ello se ha elegido una muestra de amplio espectro a nivel europeo integrada por personas mayores jubiladas. Este criterio de selección se justifica por distintos motivos. Por un lado, por la necesidad de disponer de una muestra homogénea en cuanto a disponibilidad de tiempo para participar en este tipo de actividad. Por otro, porque este trabajo parte de la hipótesis de que la motivación de los individuos al voluntariado es dinámica puesto que varía a lo largo del tiempo, así como en diferentes situaciones (Clary et al., 1998; Yeung, 2004). La escala de valores que sustentan la naturaleza humana de una persona mayor jubilada seguramente difiere del que constituye la esencia de un joven estudiante, un profesional maduro o el de un ama de casa. A medida que las personas se hacen mayores, suelen estar más involucradas en un entramado de redes sociales y más comprometidas con los patrones de vida habituales, al mismo tiempo que tratan de evitar los cambios (Tyler \& Shuller, 1991). Esta circunstancia presupone que las personas mayores correlacionen positivamente con los valores de la dimensión conservación (tradición, conformidad y seguridad) y 
negativamente con los de la dimensión apertura al cambio (estimulación, autodirección y hedonismo). $\mathrm{Al}$ mismo tiempo, los jubilados carecen de preocupaciones laborales y, muchos de ellos, tienen su vida resuelta, lo que quizás les induzca a pensar menos en su beneficio particular y más en la dicha de los demás. Por todo ello sería de esperar que los mayores retirados del mercado laboral desarrollen en mayor grado los valores de autotrascendencia (benevolencia y universalismo), relegando a un segundo plano los de autopromoción (poder, logro y hedonismo).

Dado el papel relevante que juega el sustrato de los valores en la floración del voluntariado, el objetivo primario de este trabajo es tratar de explicar desde el punto de vista de la escala de valores personales de Schwartz (1994) por qué algunas personas mayores participan en actividades de voluntariado mientras que otras no lo hacen. Los resultados obtenidos revelan que los voluntarios jubilados presentan mayor sentido de la autotrascendencia y predisposición hacia el cambio, a la vez que denotan más aversión hacia la conservación.

Estas diferencias entre grupos de individuos hacen pensar que la gestión de los valores requiere de una confección a medida, que rehúya el tan socorrido prêt-à-porter, ya que cada colectivo de voluntarios enmascara unas creencias y valores privativos, cuando no antagónicos. Conscientes de la trascendencia que este hecho puede implicar a nivel práctico, mediante un modelo de regresión logística se han puesto de manifiesto en términos probabilísticos los rasgos que caracterizan a los voluntarios mayores y jubilados en oposición a aquellas personas del mismo colectivo que no dedican tiempo a esta actividad. En este sentido el análisis multivariante evidencia que dedicarse al voluntariado está fuertemente condicionado por el conjunto de valores que identifican la esencia de la persona mayor jubilada. De esta manera, la exploración de las odds ratios desenmascara que los voluntarios mayores son mucho más benevolentes que aquellos que no están implicados en estas actividades, lo que confirma el planteamiento realizado en la primera de las hipótesis de investigación. Este resultado se encuentra en sintonía con los obtenidos en los trabajos de Pepper et al. (2009), Bathini y Vohra (2013) o el propio Schwartz (2010), en la línea de que aquellos individuos que tienen interiorizados estos valores desarrollan una conducta prosocial, una tendencia natural a comprender, aceptar y preocuparse por el bienestar del prójimo. Esta circunstancia no es de extrañar dado los valores que subyacen en el espíritu de estas personas: honestidad, lealtad, responsabilidad, sentido vital, auténtica amistad, utilidad, espiritualidad...

Dada la estructura circular de valores motivacionales opuestos o compatibles que dispone la Teoría de los valores humanos de Schwartz, en el otro extremo del eje se plantea que los valores de autopromoción -poder, logro y hedonismo- deberían asociarse negativamente con el voluntariado. 
Desde este enfoque, diferentes investigaciones han obtenido evidencias empíricas de que el deseo de autopromoción personal frena las conductas altruistas (Pepper et al., 2009, o Grönlund, 2011, 2012, entre otras). Los resultados del modelo de regresión logística revelan que el afán de poder obstaculiza el voluntariado, más que promoverlo, algo natural dados los valores dominantes que lo conforman: reconocimiento social, autoridad, riqueza, preocupación por preservar la imagen pública... Las motivaciones egoístas que hacen que el poder paralice la actividad voluntaria no se manifiestan sin embargo en relación con el hedonismo, quizás porque tal y como se comentó anteriormente, el placer como fin supremo es un valor que se encuentra a caballo entre dos dimensiones diferentes, la autopromoción y la apertura al cambio. Esta dualidad sugiere el cumplimiento parcial de la segunda hipótesis de investigación de este trabajo.

Los resultados obtenidos parecen confirmar que los tres valores que definen la dimensión apertura al cambio - estimulación, autodirección y hedonismo- constituyen predictores esenciales del voluntariado. En sintonía con los trabajos de Bathini y Vohra (2013), Mlčák y Záškodná (2014) o Randle y Dolnicar (2015), la presente investigación descubre que los jubilados más predispuestos a la experimentación y que acentúan la acción y el pensamiento libre e independiente se implican en mayor medida en tareas de voluntariado. Algunos de los rasgos característicos que incitan a estos sujetos hacia el voluntariado son el deseo de libertad, la curiosidad, la elección de objetivos propios, la independencia o la creatividad (autodirección), la variedad, emoción o atrevimiento (estimulación) o el placer, auto-indulgencia o disfrute de la vida (hedonismo). Sin duda, la apertura al cambio incentiva el voluntariado, lo que ratifica el planteamiento de la tercera hipótesis de investigación.

Por lo que respecta al eje conservación, el propio Schwartz (2010) proponía que esta dimensión exalta la autoregulación, el orden y la resistencia al cambio, lo que debería traducirse en tasas de voluntariado más bajas entre los jubilados imbuidos por estos sentimientos. De los tres valores integrantes de esta dimensión -seguridad, conformidad y tradición-, el modelo de regresión logística sólo identifica al primero de ellos como factor asociado negativamente al voluntariado, de tal manera que los mayores jubilados a los que les preocupan valores como la seguridad nacional y de sus propias familias, el orden social, la limpieza, o la salud son menos propensos a colaborar como voluntarios. Este resultado confirma la cuarta hipótesis de investigación del presente trabajo.

A modo de resumen, y en términos de dimensiones, se puede concluir que la apertura al cambio y la autotrascendencia constituyen un motor importante para propulsar la participación de los mayores jubilados en tareas de voluntariado; todo lo contrario a lo que ocurre entre aquellos individuos que 
persiguen con ahínco la autopromoción y/o la conservación, quienes por lo general rehúyen navegar en las aguas, muchas veces no cartografiadas, del voluntariado en organizaciones del tercer sector.

Con independencia de la importancia de los resultados de la investigación presentados en este estudio, estos deben ser considerados con prudencia, teniendo en cuenta las limitaciones metodológicas inherentes a él. En primer lugar, los valores han sido medidos mediante autopercepción lo que puede provocar desviaciones sesgadas respecto a lo esperado si se utilizasen variables de naturaleza objetiva. En segundo lugar, un problema metodológico relacionado podría ser el atractivo social; la influencia del atractivo en estudios sobre el voluntariado ha sido analizado por investigadores anteriores. En tercer lugar, la relación causal entre el voluntariado y los valores observados en nuestro estudio debe ser relativizada, dado que los datos del estudio son de carácter transversal y no experimental. Por lo tanto, no se pueden presuponer potenciales cambios generalizables en el comportamiento del voluntariado.

\section{Implicaciones y líneas futuras de investigación}

La presente investigación tiene importantes implicaciones prácticas, ya que indaga en lo más profundo del ser humano, sus sentimientos y valores más íntimos. Para mejorar el nivel de compromiso y la motivación de los voluntarios mayores jubilados y, de este modo, afrontar con firmeza los retos que amenazan al sector no lucrativo, la principal pauta a considerar por los responsables de recursos humanos de estas entidades es la búsqueda de coherencia entre los valores individuales y la naturaleza de la actividad que va a desempeñar el voluntario.

En definitiva, los resultados de este trabajo sugieren que la gestión del voluntariado en el tercer sector exige el diseño de políticas de recursos humanos de alto compromiso, la puesta en marcha de una praxis que contribuya a incrementar el grado de identificación de estas personas. Sólo alcanzando a conocer exactamente qué y cómo se sienten los voluntarios en el desempeño de su actividad podrán reorientarse las prácticas de gestión con vistas a la obtención de resultados sostenibles en el tiempo.

Finalmente, cabe mencionar que los cuestionarios administrados consideran el voluntariado como un fenómeno único e indiscriminado; sin embargo, es probable que el tipo de actividad constituya un factor clave a la hora de activar o desactivar el "gen" del voluntariado en las personas mayores jubiladas. Analizar la relación que existe entre diferentes tipos de voluntariado -cultural, religioso, caritativo, deportivo, etc. - y los valores personales constituye una futura línea de investigación a desarrollar en próximos trabajos. Igualmente, en el futuro podrían introducirse en el estudio algunas variables de 
control que permitieran profundizar aún más en el perfil de los voluntarios y en la influencia de los valores en el voluntariado, en función de sus características sociodemográficas (género, nivel de estudios, nivel de ingresos, estado civil, etc.).

\section{Referencias}

Anshell, C., \& Gash, A. (2008). Collaborative Governance in Theory and Practice. Journal of Public Administration Research and Theory, 18(4), 543-571. https://doi.org/10.1093/jopart/mum032

Ariza-Montes, A., Roldán-Salgueiro, J.L., \& Leal-Rodríguez, A. (2015). Employee and Volunteer. An unlikely cocktail?. Nonprofit Management and Leadership. 25(3), 255-268. https://doi.org/10.1002/nml.21121

Ayensa, E.J. (2011). El Tercer Sector, una realidad heterogénea. En El sector no lucrativo de la economía social en La Rioja: Una primera aproximación (pp. 17-28). Logroño: Instituto de Estadística de La Rioja.

Bang, H. (2015). Volunteer age, job satisfaction, and intention to stay: A case of nonprofit sport organizations. Leadership \& Organization Development Journal, 36(2), 161-176. https://doi.org/10.1108/LODJ-04-2013-0052

Bathini, D.R., \& Vohra, N. (2013). The role of traits, values and self-efficacy beliefs in volunteering. In Proceedings of the 3rd LAM (2013) conference. Ahmedabad.

Bauer, T.K., Bredtmann, J., \& Schmidt, C.M. (2013) Time vs. money — The supply of voluntary labor and charitable donations across Europe. European Journal of Political Economy, 32(0), 80-94. https://doi.org/10.1016/j.ejpoleco.2013.06.006

Beckers, T., Siegers, P., \& Kuntz, A. (2012). Congruence and performance of value concepts in social research. Survey Research Methods, 6(1), 13-24.

Bekkers, R. (2005). Participation in voluntary associations: Relations with resources, personality, and political values. Political Psychology, 26(3), 439-454. https://doi.org/10.1111/j.1467-9221.2005.00425.x

Bekkers, R., \& Bowman, W. (2009). The relationship between confidence in charitable organizations and volunteering revisited. Nomprofit and Voluntary Sector Quarterly, 38(5), 884-897. https://doi.org/10.1177/0899764008324516

Burns, DJ, Reid, J, Toncar, M, Anderson, C, \& Wells, C. (2008). The effect of gender on the motivation of members of generation Y college students to volunteers', Journal of Nonprofit and Public Sector Marketing, 19(1), 99-118. https://doi.org/10.1300/J054v19n01_05 
Clary, E.G., \& Snyder, M. (1999). The motivations to volunteer theoretical and practical considerations. Current directions in psychological science, 8(5), 156-159. https://doi.org/10.1111/1467-8721.00037

Clary, E.G., Snyder, M., Ridge, R.D., Copeland, J., Stukas, A.A., \& Haugen, J. (1998). Understanding and assessing the motivations of volunteers: A functional approach. Journal of Personality and Social Psychology, 74, 1516-1530. https://doi.org/10.1037/0022-3514.74.6.1516

Cnaan, R.A., \& Goldberg-Glen, R.S. (1991). Measuring Motivation to Volunteer in Human Services. The Journal of Applied Behavioral Science, 27(3), 269-284. https://doi.org/10.1177/0021886391273003

Dekker, P., \& Halman, L. (2003). Volunteering and Values. In P. Dekker \& L. Halman (Eds.), The Values of Volunteering (pp. 1-17). Boston, MA: Springer US. https://doi.org/10.1007/978-1-4615-0145-9

Greenfield, E.A., \& Marks, N.F. (2004). Formal volunteering as a protective factor for older adults' psychological well-being. The Journals of Gerontology Series B: Psychological Sciences and Social Sciences, 59(5), S258-S264. https://doi.org/10.1093/geronb/59.5.S258

Grönlund, H. (2011) Identity and volunteering intertwined: Reflections from the values of young adults. Voluntas, International Journal of Voluntary \& Nonprofit Organizations, 22(3), 852-874. https://doi.org/10.1007/s11266-011-9184-6

Grönlund, H. (2012). Religiousness and volunteering. Searching for connections in late modernity. Nordic Journal of Religion and Society, 25(1), 47-66.

Grönlund, H., Holmes, K., Kang, C., Cnaan, R.A., Handy, F., Brudney, J.L. et al. (2011). Cultural values and volunteering: A cross-cultural comparison of students' motivation to volunteer in 13 countries. Journal of Academic Ethics, 2, 87-106. https://doi.org/10.1007/s10805-011-9131-6

Hao, Y. (2008). Productive activities and psychological well-being among older adults. The Journals of Gerontology Series B: Psychological Sciences and Social Sciences, 63(2), S64-S72. https://doi.org/10.1093/geronb/63.2.S64

Haski-Leventhal, D. (2009). Altruism and volunteerism: The perceptions of altruism in four disciplines and their impact on the study of volunteerism. Journal for the Theory of Social Behaviour, 39(3), 271-299. https://doi.org/10.1111/j.1468-5914.2009.00405.x

Held, M., Muller, J., Deutsch, F., Grzechnik, E., \& Welzel, C. (2009). Values structure and dimensions: Empirical evidence from the German World Values Survey. World Values Research, 2 (3), 55-76. 
Hendricks, J., \& Cutler, S.J. (2004). Volunteerism and socioemotional selectivity in later life. The Journals of Gerontology Series B: Psychological Sciences and Social Sciences, 59(5), S251-S257. https://doi.org/10.1093/geronb/59.5.S251

Hitlin, S., \& Piliavin, J.A. (2004). Values: Reviving a dormant concept. Annual review of sociology, 30, 359-393. https://doi.org/10.1146/annurev.soc.30.012703.110640

Ho, Y.W., You, J., \& Fung, H.H. (2012). The moderating role of age in the relationship between volunteering motives and well-being. European Journal of Ageing, 9(4), 319-327. https://doi.org/10.1007/s10433-012-0245-5

Hofstede, G.H. (2001). Culture's consequences: Comparing values, behaviors, institutions and organizations across nations. Thousand Oaks: Sage.

Inglehart, R. (1997). Modernization and postmodernization: Cultural, economic, and political change in 43 societies (vol. 19). Princeton, NJ: Princeton University Press.

Keith, P.M. (2003). Interests and skills of volunteers in an ombudsman program: Opportunities for participation. The International Journal of Aging and Human Development, 57(1), 1-20. https://doi.org/10.2190/UXA7-0EE5-3WJ7-BLYL

Khalil, E.L. (2004). What is altruism?. Journal of economic psychology, 25(1), 97-123. https://doi.org/10.1016/S0167-4870(03)00075-8

Kluckhohn, C. (1951). Values and valueorientations in the theory of action. In T Parsons \& EA. Shils (Eds.), Toward a General Theory of Action (pp. 388-433). New York: Harper

Lindeman, M., \& Verkasalo, M. (2005). Measuring values with the short Schwartz's value survey. Journal of Personality Assessment,85(2), 170-178. https://doi.org/10.1207/s15327752jpa8502_09

Lum, T.Y., \& Lightfoot, E. (2005). The effects of volunteering on the physical and mental health of older people. Research on aging, 27(1), 31-55. https://doi.org/10.1177/0164027504271349

Luoh, M.C., \& Herzog, A.R. (2002). Individual consequences of volunteer and paid work in old age: Health and mortality. Journal of Health and Social Behavior, 43(4), 490-509. https://doi.org/10.2307/3090239

Martinson, M., \& Halpern, J. (2011). Ethical implications of the promotion of elder volunteerism: A critical perspective. Journal of Aging Studies, 25(4),427-435. https://doi.org/10.1016/j.jaging.2011.04.003

McKeever, B., \& Pettijohn, S. (2014). The Nonprofit Sector in Brief 2014: Public Charities, Giving, and Volunteering. Washington, DC: Urban Institute. 
Mlčák, Z., \& Záškodná, H. (2014). Selected Constructs in the Context of Volunteerism. In P. Záškodný, P. Procházka \& E. Ulrychová (Eds.), The proceedings of 3rd International e-Conference on Optimization, Education and Data Mining in Science, Engineering and Risk Management 2013. Praha: Publishing House Curriculum.

Moore, E., Warta, S., \& Erichsen, K. (2014). College Students' Volunteering: Factors Related to Current Volunteering. Volunteer Settings, and Motives for Volunteering. College Student Journal, 48(3), 386-396.

Morrow-Howell, N., Hinterlong, J., Rozario, P.A., \& Tang, F. (2003). Effects of volunteering on the well-being of older adults. The Journals of Gerontology Series B: Psychological Sciences and Social Sciences, 58(3), S137-S145. https://doi.org/10.1093/geronb/58.3.S137

Morrow-Howell, N., Hong, S.I., \& Tang, F. (2009). Who benefits from volunteering? Variations in perceived benefits. The Gerontologist, 49(1), 91-102. https://doi.org/10.1093/geront/gnp007

Musick, M.A., \& Wilson, J. (2008). Volunteers: A social profile. Indiana University Press.

Musick, M.A., Herzog, A.R., \& House, J.S. (1999). Volunteering and mortality among older adults: Findings from a national sample. The Journals of Gerontology Series B: Psychological Sciences and Social Sciences, 54(3), S173-S180. https://doi.org/10.1093/geronb/54B.3.S173

Nylund, M., \& Yeung, A.B. (2005). Vapaaehtoisuuden anti, arvot ja osallisuusmurroksessa. Teoksessa Marianne Nylund \& Anne Birgitta Yeung (toim.), Vapaaebtoistoiminta. Anti, arvot ja osallisuus (pp 13-38). Tampere: Vastapaino.

Okun, M.A., \& Schultz, A. (2003). Age and motives for volunteering: testing hypotheses derived from socioemotional selectivity theory. Psychology and aging, 18(2), 231. https://doi.org/10.1037/08827974.18.2.231

Okun, M.A., Barr, A., \& Herzog, A. (1998). Motivation to volunteer by older adults: A test of competing measurement models. Psychology and aging, 13(4), 608-621. https://doi.org/10.1037/08827974.13.4.608

Pepper, M., Jackson, T., \& Uzzell, D. (2009). An examination of the values that motivate socially conscious and frugal consumer behaviours. International Journal of Consumer Studies, 33(2), 126-136. https://doi.org/10.1111/j.1470-6431.2009.00753.x

Piliavin, J.A., \& Charng, H.W. (1990). Altruism: A Review of Recent Theory and Research. Annual Review of Sociology, 16, 27-65. https://doi.org/10.1146/annurev.so.16.080190.000331 
Plagnol, A., \& Huppert, F.A. (2010). Happy to help? Exploring the factors associated with variations in rates of volunteering across Europe. Social Indicators Research, 97(2), 157-176. https://doi.org/10.1007/s11205-009-9494-x

Principi, A., Chiatti, C., \& Lamura, G. (2012). Motivations of older volunteers in three European countries. International Journal of Manpower, 33(6), 704-722. https://doi.org/10.1108/01437721211261831

Puohiniemi, M. (2006). Täsmäelämän ja uusyhteisöllisyydenaika. Vantaa: Limorkustannus.

Putnam, R.D. (2000). Bowling Alone: The Collapse and Revival of American Community. New York : Simon \& Schuster. https://doi.org/10.1145/358916.361990

Randle, M., \& Dolnicar, S. (2015). The characteristics of potential environmental volunteers: Implications for marketing communications. Australasian Journal of Environmental Management, 22(3), 1-11. https://doi.org/10.1080/14486563.2014.976848

Reed, P.B., \& Selbee, K. (2003). Do people who volunteer have a distinctive ethos? A Canadian study. In P Dekker \& L Halman (Eds.), The values of volunteering: cross-cultural perspectives. New York: Kluwer Academic/Plenum Publishers. https://doi.org/10.1007/978-1-4615-0145-9_6

Rokeach M. (1973). The Nature of Human Values. New York: Free Press.

Roszkowski, M.J., Kinzler, R.J., \& Kane, J. (2014). Profile of a Residential Learning Community Dedicated to Service-Learning on Schwartz's Typology of Values. Journal of Service-Learning in Higher Education, 3, 5-29.

Schwartz, S.H. (1992). Universals in the content and structure of values: Theoretical advances and empirical tests in 20 countries. Advances in experimental social psychology, 25(1), 1-65. https://doi.org/10.1016/S0065-2601(08)60281-6

Schwartz, S.H. (1994). Are there universal aspects in the structure and contents of human values?. Journal of social issues, 50(4), 19-45. https://doi.org/10.1111/j.1540-4560.1994.tb01196.x

Schwartz, S.H. (2006). A theory of cultural value orientations: Explication and applications. Comparative sociology, 5(2), 137-182. https://doi.org/10.1163/156913306778667357

Schwartz, S.H. (2007). Value orientations: Measurement, antecedents, and consequences across nations. Measuring attitudes cross-nationally: Lessons from the European Social Survey (pp. 161-193). London: Sage. https://doi.org/10.4135/9781849209458.n9 
Schwartz, S.H. (2010). Basic values: How they motivate and inhibit prosocial behavior. In M. Mikulincer \& P.R. Shaver (Eds.), Prosocial motives, emotions, and behavior: The better angels of our nature. (pp. 221-241). Washington: American Psychological Association. https://doi.org/10.1037/12061-012

Smith, D.H. (2000). Grassroots Associations. Thousand Oaks, CA: Sage.

Snyder, M., \& Omoto, A.M. (1992). Who helps and why? The psychology of AIDS volunteerism. In S. Spacapan \& S. Oskamp (Eds.), Helping and being belped: Naturalistic studies (pp. 213-239). Newbury Park, C.A.: SAGE.

Sprecher, S. \& Fehr, B. (2005). Compassionate love for close others and humanity. Journal of Social and Personal Relationships, 22, 629-652. https://doi.org/10.1177/0265407505056439

Tang, F., Choi, E., \& Morrow-Howell, N. (2010). Organizational support and volunteering benefits for older adults. The Gerontologist, 50(5), 603-612. https://doi.org/10.1093/geront/gnq020

Tyler, T.R. \& Shuller, R.A (1991). Aging and attitude change. Journal of Personality and Social Psychology, 61, 689-697. https://doi.org/10.1037/0022-3514.61.5.689

Unger, L.S. (1991). Altruism as a motivation to volunteer. Journal of Economic Psychology, 12(1), 71-100. https://doi.org/10.1016/0167-4870(91)90044-T

Van Willigen, M. (2000). Differential benefits of volunteering across the life course. The Journals of Gerontology Series B: Psychological Sciences and Social Sciences, 55(5), 308-318. https://doi.org/10.1093/geronb/55.5.S308

Wilson, J. (2000) Volunteering. Annual Review of Sociology, 26, 215-240. https://doi.org/10.1146/annurev.soc.26.1.215

Yeung, A.B. (2004). The octagon model of volunteer motivation: Results of a phenomenological analysis. Voluntas: International Journal of Voluntary \& Nonprofit Organizations, 15, 21-46. https://doi.org/10.1023/B:VOLU.0000023632.89728.ff

Intangible Capital, 2017 (www.intangiblecapital.org)

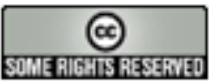

Article's contents are provided on an Attribution-Non Commercial 3.0 Creative commons license. Readers are allowed to copy, distribute and communicate article's contents, provided the author's and Intangible Capital's names are included. It must not be used for commercial purposes. To see the complete license contents, please visit http://creativecommons.org/licenses/by-nc/3.0/. 